\title{
Diversité Et Dynamique Des Communautés De Vers De Terre De Trois Formations Végétales Dans Une Savane Humide De l'Afrique De l'Ouest (Lamto, Côte d'Ivoire)
}

\author{
Soro Nounfro Madjima, \\ Ehouman N'guetta Moïse, \\ Toure Mamadou,
} Unité de Recherche en Ecologie et Biodiversité (UREB), UFR des Sciences de la Nature (SN), Université Nangui Abrogoua, Abidjan, Côte d'Ivoire

\section{Ouattara Noufou Doudjo,}

Unité de Recherche en Ecologie et Biodiversité (UREB), UFR des Sciences de la Nature (SN), Université Nangui Abrogoua, Abidjan, Côte d'Ivoire,

Centre Suisse de Recherche Scientifique en Côte d'Ivoire,

Abidjan, Côte d'Ivoire

Tiho Seydou,

Unité de Recherche en Ecologie et Biodiversité (UREB), UFR des Sciences de la Nature (SN), Université Nangui Abrogoua, Abidjan, Côte d'Ivoire

Doi: 10.19044/esj.2018.v14n27p281 URL:http://dx.doi.org/10.19044/esj.2018.v14n27p281

Abstract

The maintenance and stability of many terrestrial ecosystems are in part related to the ecosystem functions and services of soil organisms. The present study aims to evaluate the influence of litter biomass produced by three types of vegetation in the Lamto reserve on the dynamics of earthworm community. Each vegetation formations has been subdivided into 3 parcels. These parcels were geo-referenced using a GPS and mapped using QGIS software. For each plot, 25 points were randomly selected using their GPS coordinates. The litters were collected by manual collection to evaluate their biomass. Monoliths of the TSBF (Tropical Soil Biology) type were dug in order to collect the earthworms by direct manual sorting. Also, soil clumps were collected to determine the organic matter levels by the method of lossto-fire. In total, 12 species of earthworms belonging to 3 ecological categories were collected. The forest block had the highest density of earthworms with a predominance of epigeic earthworms followed by grassy savannah with a predominance of endogeic earthworms. The wooded savannah has the lowest density of earthworms with a predominance of anecic earthworms. In terms 
of species richness, the grassy savannah contained the greatest number of earthworm species followed by the wooded savannah and the forest block that had the same number of species. In the three vegetations formations, it was found that earthworm densities were correlated with litter biomass as well as organic matter levels (correlation between earthworm density and litter biomass: $r^{2}=0,75$; correlation between density of earthworms and the rate of organic matter: $\left.r^{2}=0,93\right)$.

Keywords: Earthworms, Litter, Vegetations formations, Lamto reserve, Côte d'Ivoire

\section{Résumé}

Le maintien et la stabilité de nombreux écosystèmes terrestres sont en partie liés aux fonctions et services écosystémiques des organismes du sol. La présente étude vise à évaluer l'influence de la biomasse de litière produite par trois types de formations végétales de la réserve scientifique de Lamto sur la dynamique des communautés de vers de terre. Chaque formation végétale a été subdivisée en 3 parcelles. Celles-ci ont été géo-référencées à l'aide d'un GPS et cartographiées à l'aide du logiciel QGIS. Pour chaque parcelle, 25 points ont été choisis de façon aléatoire grâce à leurs coordonnées GPS. Les litières ont été prélevées par ramassage manuel afin d'évaluer leur biomasse. Des monolithes de type TSBF (Tropical Soil Biology) ont été creusés afin de collecter les vers de terre par tri manuel direct. Aussi, des mottes de terre ont été prélevées afin de déterminer les taux de matière organique par la méthode de perte au feu. Au total, 12 espèces de vers de terre appartenant à 3 catégories écologiques ont été collectées.L'îlot forestier a présenté la plus forte densité de vers de terre avec une prédominance des vers épigés suivi de la savane herbeuse avec une dominance des vers endogés. La savane arborée a présenté la plus faible densité de vers de terre avec une prédominance des vers anéciques. Pour ce qui est de la richesse spécifique, la savane herbeuse renfermait le plus grand nombre d'espèces de vers de terre suivie de la savane arborée et de l'îlot forestier qui avaient eux le même nombre d'espèces. Il a été constaté au sein des 3 formations végétales, que les densités de vers de terre étaient corrélées aussi bien aux biomasses des litières qu'aux taux de matière organique (corrélation entre la densité des vers de terre et la biomasse de la litière: $r^{2}=0,75$, corrélation entre la densité des vers de terre et le taux de matière organique : $r^{2}=0,93$ ).

Mots-clés: Vers de terre, Litières, Formations végétales, Réserve de Lamto, Côte d'Ivoire 


\section{Introduction}

La faune du sol joue un rôle primordial dans l'incorporation et la transformation des matières ainsi que l'amélioration de la structure et des propriétés physico-chimique du sol (Brussaard, 2012). Ainsi, cette faune améliore la fertilité des sols qui est fortement liée à la quantité et la qualité des matières transformées au cours de leurs différentes activités (Gobat etal., 2010 ; Blouin et al., 2013). Parmi les organismes qui constituent cette faune, les vers de terre représentent l'une des composantes majeures puisque, dans la plupart des écosystèmes terrestres, ils dominent en biomasse (Gobat et al., 2003 ; Pelosi, 2008). Par ailleurs, leurs activités dans divers types d'écosystèmes sont caractérisées par leur grande capacité à incorporer au sol de la litière végétale. Cette litière est soit consommée directement en surface, soit après avoir été enfouie dans les galeries (Blouin et al., 2013 ; Brussaard et al., 2007). Ainsi, les vers de terre ingèrent divers types de matières, qui diffèrent en quantité et en qualité selon l'hétérogénéité du paysage (Jégou et al., 2000 ; Amossé et al., 2015). La variabilité spatiale de ces matières à différents niveaux dans le sol conduit les vers de terre à adopter divers modes d'alimentation. Trois catégories ont été définies par Bouche (1977) : les vers épigés (pigmentés) qui vivent et se nourrissent dans la litière de surface audessus des sols minéraux, les vers anéciques (pigmentés) qui s'enfouissent dans le sol et se nourrissent de la litière de surface et les vers endogés (non pigmentés) qui se déplacent près de la surface du sol mais, se nourrissent de la litière partiellement décomposée et de la matière organique du sol. De ce fait, les vers de terre influencent considérablement les propriétés physicochimiques et biologiques du sol (Doan et al., 2013, Singh et al., 2016). Leur diversité est un facteur clé pour la stabilité des écosystèmes, responsable des services écosystémiques nécessaires à l'épanouissement de l'homme (Eisenhauer et Schädler, 2011; Blouin et al., 2013 ; Dewi et Senge, 2015). Par ailleurs, de nombreux auteurs ont révélé que la lutte contre la dégradation des sols, la conservation de la biodiversité épigée et endogée ainsi que des services écosystémiques associés tels que la décomposition, le cycle des éléments nutritifs, la séquestration du carbone, la détoxification et le maintien des propriétés physico-chimiques du sol, sont déterminés par la quantité de litière (Lavelle et Spain, 2011 ; Pérès et al., 2011). Pourtant, très peu d'études ont porté sur la dynamique des communautés de vers de terre en fonction des biomasses de litières produites dans différentes formations végétales des écosystèmes tropicaux. La présente étude vise à évaluer la diversité et la dynamique des vers deterre de trois habitats (savane herbeuse, savane arborée, îlot forestier) de la réserve de Lamto. Cette étude vérifie l'hypothèse que la diversité et la dynamique des vers de terre seraient fortement influencées par les biomasses de litière produites dans les différents types de formation végétale. 


\section{Matériel et méthodes:}

\section{Site d'étude:}

Cette étude a été réalisée dans la réserve naturelle de Lamto en Côte d'Ivoire $\left(6^{\circ} 13 \mathrm{~N}, 5^{\circ} 2 \mathrm{~W}\right)$ située à $160 \mathrm{~km}$ au Nord-Ouest d'Abidjan. Cetteréserve est localisée dans une zone de transition entre la forêt humide semi-décidue au Sud et les savanes soudaniennes au Nord. Avec une superficie de 2700 ha, la réserve de Lamto se caractérise par une pluviométrie bimodale indiquant deux saisons pluvieuses d'avril à juillet et de septembre à octobre puis deux saisons sèches dont la petite saison qui a lieu en août et la grande saison allant de novembre à mars. La température annuelle moyenne sur les 10 dernières années (2007-2017) était de $28,21^{\circ} \mathrm{C}$ et la pluviométrie annuelle moyenne est de $1240,92 \mathrm{~mm}$. La plupart des sols reposent sur des roches granitiques. Cette réserve compte 7 faciès de végétation (Cesar et Menaut, 1974). Dans le cadre de cette étude seuls trois faciès, les plus représentatifs de cette savane ont été échantillonnés. Il s'agit de la savane herbeuse, composée essentiellement de graminées, de la savane arborée ayant une végétation hétérogène composée de graminées et d'arbustes et d'un îlot forestier constitué principalement d'arbres.

\section{Dispositif d'échantillonnage:}

Chacune des formations végétales (savane herbeuse, savane arborée et îlot forestier) a été subdivisée en 3 parcelles d'échantillonnage. Ces parcelles d'échantillonnages ont d'abord été géo-référencé à l'aide d'un GPS de marque GARMIN Montana 600. Les données de géo-référence ont été utilisées pour générer les cartes des différentes parcelles à l'aide du logiciel QGIS2.6.0 (Figure 1). Au sein de chaque parcelle, 25 carrésde $5 \mathrm{~m}$ de côté ont été choisis de façon aléatoire à partir de leurs coordonnées géographiques afin de collecter la litière, les vers de terre et des mottes de sols (Figure 1). 


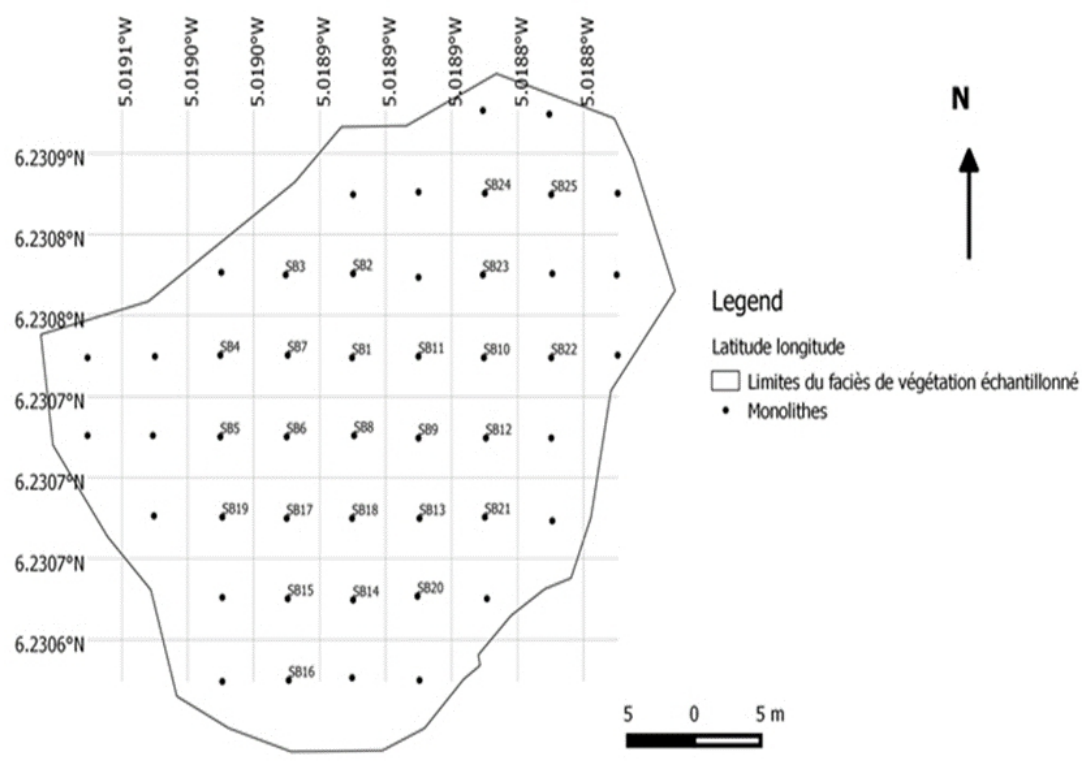

Figure 1: Dispositif d'une parcelle d'échantillonnage de la savane herbeuse à titre d'exemple.

\section{Collecte des litières, des vers de terre et des mottes de sol dans les faciès de végétation:}

Dans chaque type de végétation, la litière a été ramassée dans 25 carrés de $5 \mathrm{~m}$ de coté manuellement et placée dans du papier journal dûment étiqueté (date, nom de la parcelle, numéros du carré). Après la collecte de la litière, des monolithes de type TSBF (Tropical Soil Biology) modifié $(50 \mathrm{~cm}$ x $50 \mathrm{~cm}$ x $30 \mathrm{~cm}$ ) ont été creusés à l'intersection des principales diagonales des différents carrés dans lesquels une fouille manuelle systématique a été opérée afin de récolter tous les individus de vers de terre présents. Cette fouille a été menée de manière stratifiée $(0-10 \mathrm{~cm} ; 10-20 \mathrm{~cm}$ et $20-30 \mathrm{~cm})$, selon la technique proposée par Lavelle (1978). Par ailleurs, un échantillon de sol des différentes parcelles d'échantillonnage de chaque formation végétale a été également prélevé à partir des monolithes et conservés dans des boîtes dûment étiquetées puis acheminées au laboratoire.

\section{Détermination de la biomasse des litières et identification des vers de terre:}

Afin de déterminer leurs biomasses, les litières prélevées et acheminées au laboratoire ont été séchées à l'étuve à $105^{\circ} \mathrm{C}$ pendant 48 heures. Les masses des matières sèches obtenues ont été mesurées à l'aide d'une balance de type Sartorius de précision 0,1.

Les vers de terre collectés ont d'abord été conservés dans des bocaux dûment étiquetés contenant du formol dilué à $4 \%$. Ensuite, à l'aide d'une 
loupe binoculaire, l’identification des espèces a été réalisée à partir des clés de détermination de Cszudi et Tondoh (2007), basées sur la morphologie (forme, taille, longueur des segments) et la pigmentation des individus.

\section{Détermination de la teneur en matière organique du sol (MOS):}

La teneur en matière organique du sol des parcelles de chacune des formations végétales échantillonnées a été mesurée à partir des échantillons composites de sol prélevés au niveau de chaque monolithe creusé. Ainsi, les sols prélevés ont d'abord été séchés à l'étuve pendant 24 heures à $105^{\circ} \mathrm{C}$. Ensuite, ils ont été tamisés et $5 \mathrm{~g}$ ont été brûlés au four analyseur de marque LECO CNS 2000 (LECO corporation, St Joseph, MI) à une température de $375^{\circ} \mathrm{C}$ pendant 16 heures. Ces échantillons de sol ont été pesés à nouveau afin de déterminer leurs poids brûlés (Hasine et al., 2008). Le taux de matière organique a été déterminé selon la formule suivante:

Où

$$
\% \text { MOS }=\frac{(\text { PNB }- \text { PB })}{\text { PNB }} \times 100
$$

PNB = Poids du sol non brûlé et $\mathbf{P B}=$ poids dusol brûlé

MOS = matière organique du sol

\section{Analyses statistiques:}

Les densités moyennes des vers de terre des différentes formations ont été comparées à l'aide du test non paramétrique de Kruskal-Wallis suivi du test de Kolmogorov-Smirnov grâce au logiciel STATISTICA 7.1. Les indices de diversité ont été déterminés à partir du calcul des indices de diversité de Shannon-Wiever $(\mathrm{H})$ et de l'équitabilité à l'aide du logiciel EstimateS 7.5. Le logiciel XLSTAT a été utilisé pour déterminer les coéfficients de corrélations entre la densité des vers de terre et la biomasse des litière d'une part et entre la densité des vers de terre et le taux de matière organique d'autre part.

\section{Résultats:}

\section{Densités des vers de terre collectés dans les formations végétales:}

$\mathrm{Au}$ total, 12 espèces de vers de terre ont été collectées au sein des 3 formations végétales échantillonnées. Ces espèces appartiennent principalement à 3 catégories écologiques que sont les vers anéciques, les vers épigés et les vers endogés (Tableau 1).Il ressort que la densité moyenne des vers de terre collectés dans l'îlot forestier est la plus élevée (172 $\pm 213,77$ $\mathrm{ind} / \mathrm{m}^{2}$ ) suivie par la savane herbeuse $\left(137,60 \pm 58,70 \mathrm{ind} / \mathrm{m}^{2}\right)$ et la savane arborée $\left(102,61 \pm 123,81 \mathrm{ind} / \mathrm{m}^{2}\right)$. La comparaison des densités moyennes de vers de terre des différentes formations végétales révèle l'existence de différences significatives entre celles-ci (test de Kruskal -Wallis:F = 15, 05 P 
$<0,005)$. Le test de Kolmogorov-Smirnov montre une différence significative entre la savane herbeuse et la savane boisée ;entre la savane herbeuse et la forêt puis entre la savane boisée et la forêt ( $\mathrm{P}<0,005$ pour chacun).

Chaque type de formations végétales est rattachée une catégorie écologique. Ainsi, la densité moyenne des vers anéciques est plus élevéeen savane arborée $\left(35,2 \pm 26,38 \mathrm{ind} / \mathrm{m}^{2}\right)$. Dans l'îlot forestier celle des vers épigés est plus élevée $\left(86,56 \pm 59,11 \mathrm{ind} / \mathrm{m}^{2}\right)$ et en savane herbeuse les endogées dominent $\left(104,16 \pm 70,57 \mathrm{ind} / \mathrm{m}^{2}\right)$ (Tableau 1).

La comparaison des densités moyennes des catégories écologiques de vers de terre des différentes formations végétales révèle l'existence de différences significatives entre celles-ci (test de Kruskal-Wallis: F = 17, 26;P $<0,005)$. Le test de Kolmogorov-Smirnov montre une différence significative entre les vers épigés et les vers anéciques ; entre les vers anéciques et les vers endogés et entre les vers épigés et les vers endogés ( $\mathrm{P}<0,005$ pour chacun).

Tableau 1 : Densité des vers de terre (ind. $/ \mathrm{m}^{2}$ ) au sein des faciès échantillonnés

\begin{tabular}{lcccc} 
Espèces & $\begin{array}{c}\text { Catégories } \\
\text { Ecologiques }\end{array}$ & $\begin{array}{c}\text { Savane } \\
\text { herbeuse }\end{array}$ & $\begin{array}{c}\text { Savane } \\
\text { arborée }\end{array}$ & $\begin{array}{c}\text { îlot } \\
\text { forestier }\end{array}$ \\
\hline Agastrodrilus multivesiculatus & Endogé & $0,96 \pm 2,09$ & $1,28 \pm 0,09$ & 0 \\
Chuniodrilus palustris & Endogé & $27,04 \pm 08,71$ & $7,16 \pm 1,02$ & $12,8 \pm 05,70$ \\
Chuniodrilus zielae & Endogé & $1,28 \pm 0,49$ & $0,64 \pm 0,01$ & $0,96 \pm 0,09$ \\
Dichogaster agilis & Epigé & $5,44 \pm 5,52$ & $25,28 \pm 4,15$ & $72,96 \pm 6,43$ \\
Dichogaster baeri & Epigé & $0,32 \pm 0,06$ & $0,32 \pm 0,01$ & $13.44 \pm 1,88$ \\
Dichogaster saliens & Anécique & $0,64 \pm 0,79$ & $4,12 \pm 0,41$ & $0,96 \pm 0,04$ \\
Dichogaster terrae nigrae & Anécique & $0,16 \pm 0,02$ & 0 & 0 \\
Hyperiodrilus africanus & Anécique & $0,64 \pm 0,05$ & $2,76 \pm 0,21$ & $10,4 \pm 3,86$ \\
Millsonia ghanensis & Endogé & $0,16 \pm 0,06$ & 0 & $0,16 \pm 0,01$ \\
Millsonia lamtoiana & Epigé & $0,48 \pm 0,02$ & $0,48 \pm 0,01$ & $0,16 \pm 0,00$ \\
Millsonia omodeoi & Anécique & $18,08 \pm 1,00$ & $28,32 \pm 1,44$ & $5,12 \pm 1,04$ \\
Sthulmania porifera & Endogé & $74,72 \pm 12,97$ & $22,76 \pm 4,85$ & $20,80 \pm 2,87$ \\
Densité totale & - & $137,6 \pm 38,70$ & $102,61 \pm 43,81$ & $172 \pm 33,77$ \\
Densité totale des anéciques & - & $19,52 \pm 3,19$ & $35,20 \pm 6,38$ & $16,48 \pm 1,04$ \\
Densité Totale des épigés & - & $6,24 \pm 0,44$ & $26,08 \pm 2,59$ & $86.56 \pm 8,11$ \\
Densité Totale des endogés & - & $104,16 \pm 20,57$ & $31,84 \pm 1,97$ & $34,72 \pm 4,47$ \\
\hline
\end{tabular}

\section{Richesse spécifique et Indice de diversité des differentes formations végétales}

La savane herbeuse renferme le plus grand nombre d'espèce ( 12 espèces) suivie de la savane arborée et de l'îlot forestier ( 10 espèces chacun). L'indice de diversité est élévé en savane herbeuse $(\mathrm{H}=2,76)$ et faible en savane arborée et dans l'îlot forestier $(\mathrm{H}=1,87$ et $\mathrm{H}=1,85$ respectivement $)$. $\mathrm{La}$ valeur de l'équitabilité est faible en savane herbeuse ( $\mathrm{E}=0,76)$. Elle est de 0,81 pour la savane arborée et de 0,85 pour l'îlot forestier ( Tableau 2). 
Tableau 2: Nombre d'espèces,Indice de diversité de Shannon-Wiever, équitabilité

Paramètres de diversité

Savane herbeuse

Savane arborée

îlot forestier

\begin{tabular}{lccc}
\hline Indice de diversité $(\mathbf{H})$ & 2,11 & 1,87 & 1,85 \\
Equitabilité $(\mathbf{E})$ & 0,76 & 0,81 & 0,85 \\
Nombre d'espèces & 12 & 10 & 10 \\
\hline
\end{tabular}

Corrélation entre les densités de vers de terre et les biomasses de litières:

Dans les differentes formations végétales, les densités moyennes de versde terrevariaient, de 58 ind. $/ \mathrm{m}^{2}$ à 308 ind. $/ \mathrm{m}^{2}$ en savane herbeuse,de 32 ind. $/ \mathrm{m}^{2}$ à 232 ind. $/ \mathrm{m}^{2}$ en savane arborée et de 28 ind. $/ \mathrm{m}^{2}$ à 428 ind. $/ \mathrm{m}^{2}$ dans l'îlot forestier. Quant aux biomasses moyennes de litières, elles variaient de $3,33 \mathrm{~g}$ à 45,83 g en savane herbeuse, de 68,96 g à 522,76 g en savane arborée et de 67,45 g à 858,73 $\mathrm{g}$ dans l'îlot forestier.Dans l'ensemble, au sein des trois formations végétales, les points de fortes densités de vers de terre correspondent aux zones de fortes biomasses de litièreet les points de faibles densités,correspondent aux zones de faibles biomasses de litière (Figure 2A, 2B et 2C). En somme, on note une forte correlation entre la densité des vers de terre et les biomasses des litières $\left(r^{2}=0,75\right)$.

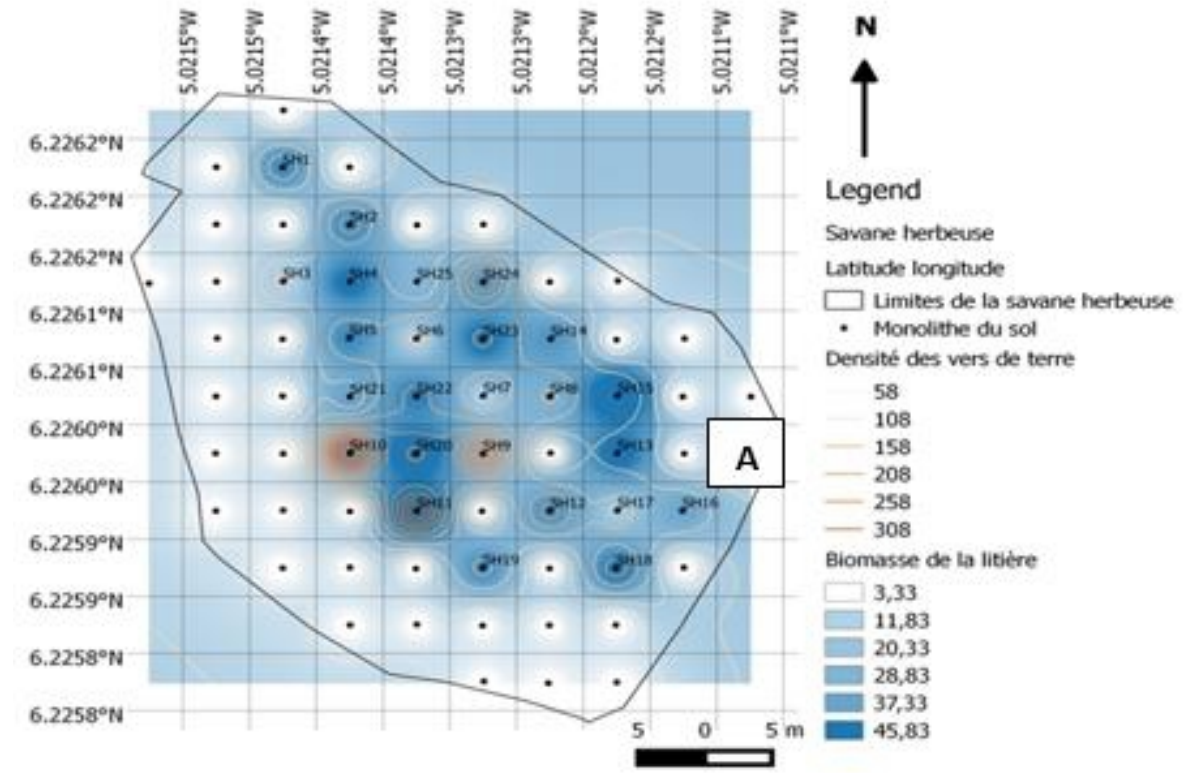



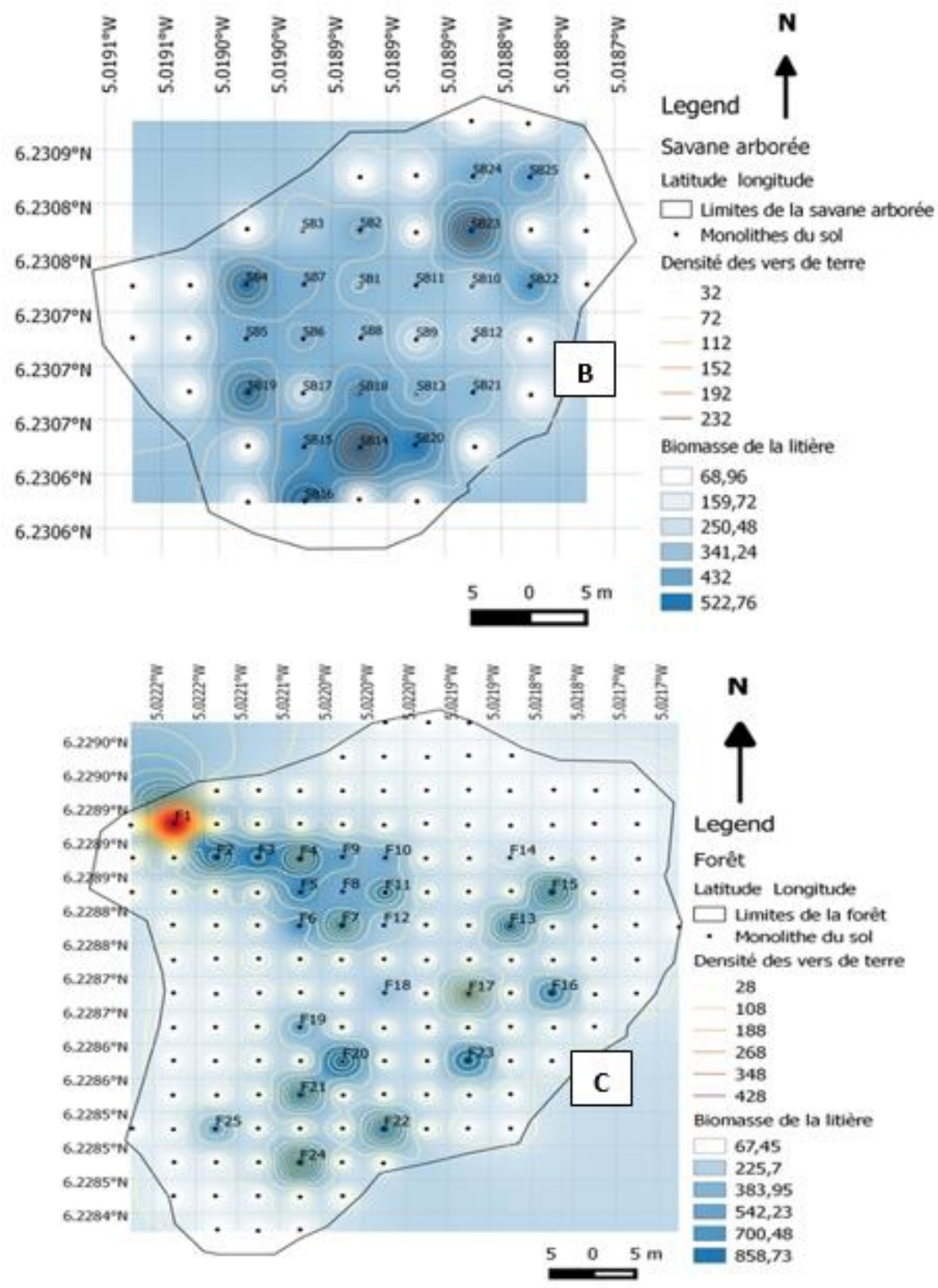

Figure 2 : Corrélation entre les communautés de vers terre (ind./m2) et les biomasses de litière (g): A : corrélation en savane herbeuse; $\mathbf{B}$ : corrélation en savane arborée; $\mathbf{C}$ : corrélation dans l'îlot forestier.

\section{Corrélation entre les densités de vers de terre et les taux de matière organique:}

Les taux moyens de matières organiques variaient de $0,59 \%$ à $6,34 \%$ en savane herbeuse, de $1,27 \%$ à $5,77 \%$ en savane arborée et de $0,70 \%$ à $14,70 \%$ dans l'îlot forestier. Aux points de forts taux de matière organique 
correspondent les fortes densités moyennes de vers de terre. Par contre aux points de faibles taux de matière organique correspondent les faibles densités moyennes de vers de terre (Figure 3A, 3B et 3C). Le test de correlation entre la densité des vers de terre et le taux de matière organiques montre qu'il existe une forte correlation entre ceux-ci $(\mathrm{r} 2=0,93)$.
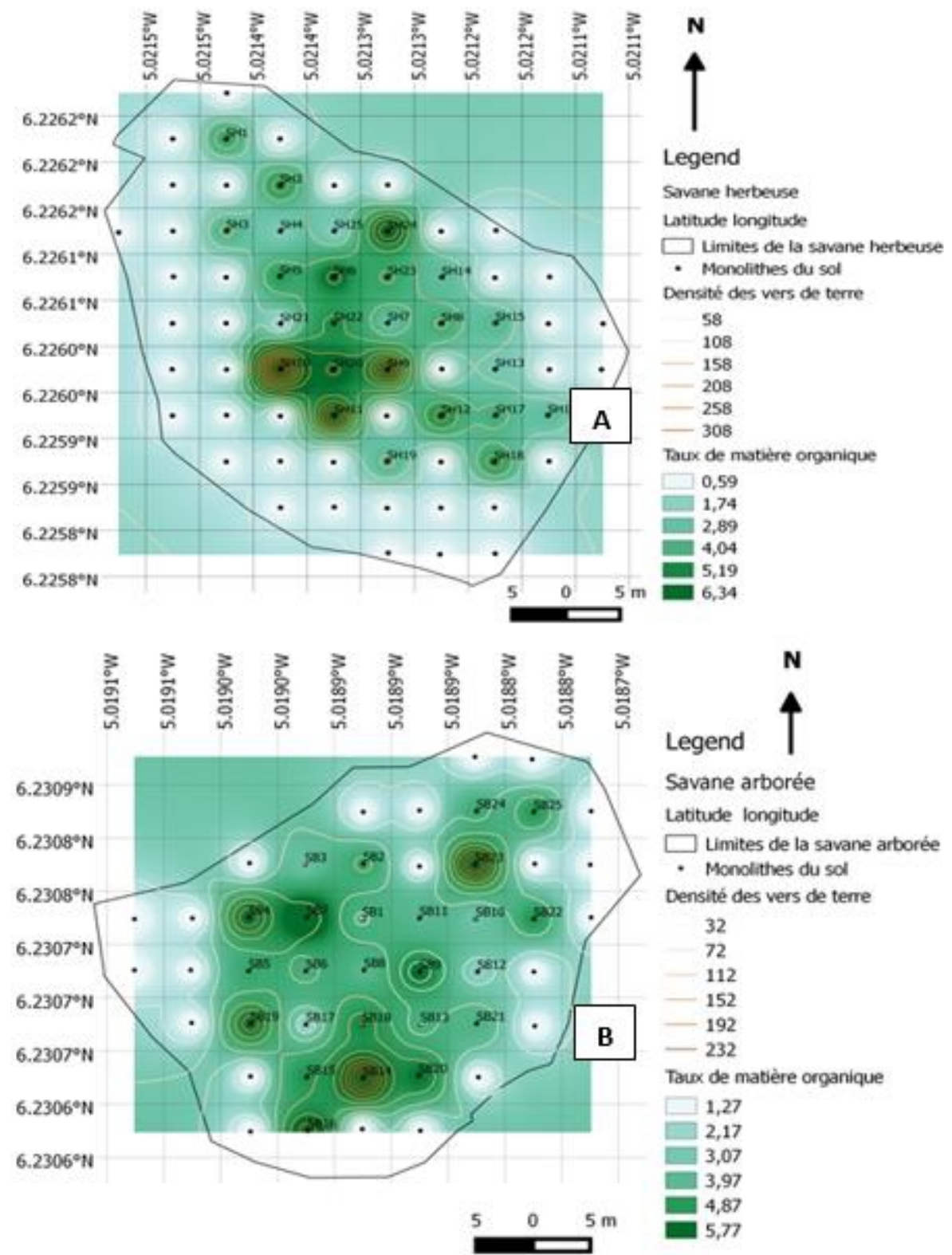


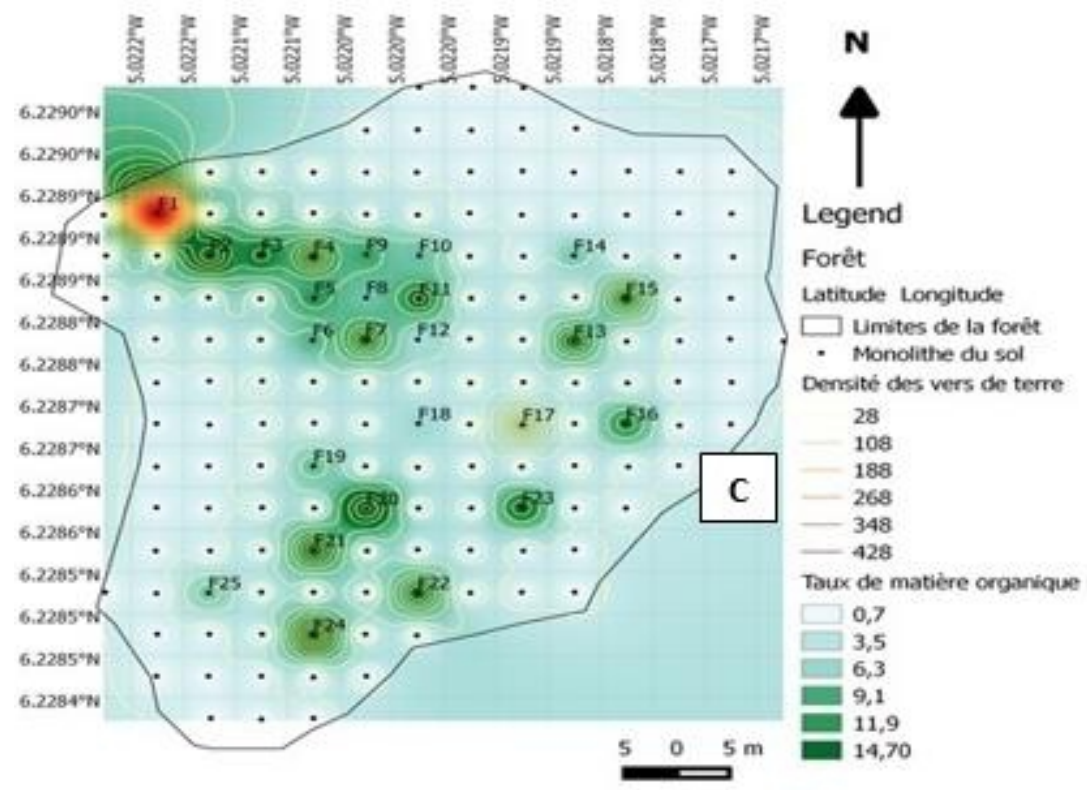

Figure 3 : Corrélation entre les communautés de vers terre (ind $/ \mathrm{m} 2)$ et les taux de matière organique (\%): A : corrélation en savane herbeuse; $\mathbf{B}$ : corrélation en savane arborée; $\mathbf{C}$ : corrélation dans l'îlot forestier.

\section{Discussion:}

La densité et le nombre d'espèce des vers de terre varient selon la formation végétale et sont fonction de la catégorie écologique. Ces résultats montrent que la survie, la fluctuation et les activités des vers de terre au sein des différentes formations végétales seraient liées aux types de litières produites et aux conditions écologiques qui y règnent. Il a été observé lors des échantillonnages que la savane herbeuse était caractérisée par la présence d'importantes graminées telles que Brachiaria brachylopha, Hyparrhenia dipandra, Imperata cylindrica et Loudetia simplex. Abbadie et al. (2006), ont montré que le système racinaire de ces graminées offre des conditions favorables aux vers de terre surtout pour l'acquisition de la nourriture. De ce fait la prédominance des vers endogés qui sont aussi les plus diversifiés pourrait se justifier par le fait qu'ils vivent dans les couches profondes et consomment principalement le sol mélangé à la litière (Bouché, 1977). La végétation en savane arborée était caractérisée par la présence des graminées (Brachiaria brachylopha, Hyparrhenia smithiana, Imperata cylindrica et Loudetia simplex) et des arbustes (Nauclea latifolia, Bridelia ferruginea, Commelina sp et Eriosema molle). Selon Ehouman et al. (2011), l'hétérogénéité de la végétation maintient un environnement favorable aux vers de terre et autres organismes du sol qui contribuent à la décomposition de la matière organique. La prédominance des vers anéciques au sein de cette formation végétale pourrait se justifier par le fait que ces espèces de vers de 
terre vivent dans les couches intermédiaires et mènent une partie de leur vie dans le sol où ils sont fortement liés aux racines des graminées. Puis, ils remontent souvent en surface pour récupérer la litière provenant des arbustes (Bouché, 1977). L'îlot forestier était principalement composé de gros arbres tels que Diospyrosmes piliformis, Erythroxylum marginatum, Ceiba pentandra, Cnectis ferruginea qui sont des arbres produisant d'importantes quantités de litières à la surface du sol. Cette litière des arbres principalement composée de feuilles a des capacités de décomposition différentes selon les espèces. La présence de cette litière offre des conditions favorables pour les vers épigés qui vivent en surface et participent à leur décomposition grâce à une relation de mutualisme établie avec des microorganismes du sol (Abbadie et al. 2006). De plus, des études menées par Satchell (1980) ont révélé que les vers épigés ont une capacité de reproduction très élevée et une croissance rapide compensant leur faible longévité (stratégie biodémographique de type r). Ceci pourrait expliquer l'importance des vers de terre épigésdans l'îlot forestier.

Par ailleurs, au sein des trois formations végétales, les densités de vers de terre ont été fortement proportionnelles aussi bien aux biomasses de litière qu'aux taux de matière organique. De plus les influences exercées par les densités de vers de terre sur les biomasses de litière et les taux de matière organique avaient des proportions différentes selon les formations végétales échantillonnées. Ces résultats traduisent de fortes implications des vers de terre dans la minéralisation des différents types de litière. Mais cette minéralisation réalisée en partie par les vers de terre serait fonction de la quantité et de la qualité de la litière produite par les différentes formations végétales et aussi leur mode d'alimentation au sein de ces biotopes. En effet, au cours de leurs activités, les vers de terre peuvent consommer et incorporer de grandes quantités de matière organique et de sol. Ce mélange est en grande partie responsable de la formation de sols meubles dans lesquels les horizons organiques de surface sont soigneusement mélangés avec le sol minéral sousjacent (Iman et Rahmani, 2005; Sautter et al., 2006). Ainsi, en savane herbeuse, la litière des feuilles produite est de petite quantité et se décompose rapidement. De ce fait, les vers endogés qui y sont prédominants se nourrissent de préférence avec les racines des herbes. Ils consomment principalement le sol et la matière organique humifiée associée dans la couche supérieure de minéraux et les exsudations de racines facilitant ainsi la décomposition (Butenschoen et al., 2009 ; Laossi et al., 2009). Selon Lavelle (1983), ces vers endogés géophages ont une capacité enzymatique digestive médiocre, tandis que la relation mutualiste entre la microflore du sol et les vers de terre peut favoriser la digestion des composés organiques. En savane arborée, la litière qui est produite provient aussi bien des plantes herbacées que des arbustes. La minéralisation decette litière hétérogène serait sous l'influence des vers 
anéciques qui se nourrissent à la surface du sol en traînant les feuilles, le fumier et d'autres matières organiques partiellement décomposées dans le sol (Römbke et $a l ., 2005$ ). Dans l'îlot forestier, la litière est présente en quantité importante et principalement constituée de feuilles de différentes espèces de plantes. Les vers épigés qui prédominent se nourrissent de la litière et/ou de la microflore attachée et ingèrent peu ou pas de sol (Römbke et $a l$., 2005). Ils peuvent fragmenter les déchets organiques en particules beaucoup plus fines en les faisant passer à travers un gésier de broyage. En raison de leurs habitudes alimentaires, l'activité de la cellulose est beaucoup plus élevée chez les vers épigés que chez les autres espèces (Römbke et $a l .$, 2005). Ces résultats montrent que dans les principales formations végétales de la réserve de Lamto (savane herbeuse, savane arborée et îlot forestier), les différentes activités des vers semblent accélérer la décomposition des litières, augmenterles taux de transformation des nutriments et d'absorption des nutriments, améliorerles propriétés physico chimiques des sols.

\section{Conclusion:}

Dans la réserve de Lamto, la densité des communautés de vers de terre est plus élevée dans les îlots forestiers mais leur richesse spécifique est plus élevée en savane herbeuse. A chaque principale formation végétale est associéé la dominance d'une catégorie écologique bien précise qui assure la minéralisation des litières produites. Pour les differents faciès de la réserve de Lamto, la transformation de la litière en matière organique au sein des principales formations végétales est en partie assurée par les communautés de vers de terre. Mais, les corrélations entre les densités de vers de terre et les biomasses de litière et entre les densités de vers de terre et les taux de matière organique diffèrent selon les formations végetales. Ces résultats montrent qu'il ya une variation du taux de minéralisation en fonction des activités et du régime alimentaire des vers de terre.

\section{Remerciements:}

Nous remercions le Professeur KoloYEO, Directeur de la Station de Recherche en Ecologie de Lamtopour le soutien matériel et logistique dans la réalisation de cette étude. Nos remerciements vont également à l'endroit du Professeur Souleymane Konaté, Directeur de l'Unité de Recherche en Ecologie et Biodiversité de l'Université NanguiAbrogoua, pour le soutien moral et matériel qu'il nous a accordé.

\section{References:}

1. Abbadie L.,Gignoux J., Lepage M. (2006). LAMTO: Structure, functioning and dynamics of a savanna ecosystem. Springer-Verlag, New York, Ecological studies vol. 179,412 pp. 
2. Amosse J., Turberg P., Kohler-Milleret R., Gobat J., Le Bayon, R.(2015). Effects of endogeic earthworms on the soil organic matter dynamics and the soil structure in urban and alluvial soil materials. Geoderma, 243-244, 50-57.

3. Blouin M., Hodson M.E., Delgado E.A., Baker G., Brussaard L., ButtK.R., Dai J., Dendooven L., Peres G., Tondoh J.E., Cluzeau D., Brun J.J. (2013). A review of earthworm impact on soil function and ecosystem services. European Journal of Soil Science, 64: 161-182.

4. Bouché M.B. (1977). Stratégies lombriciennes. In: Lohm, U., Persson, T. (Eds.), Soil Organisms as Components of Ecosystems. Ecological Bulletin, 25:122-132.

5. Brussaard L., Pulleman M.M., Ouedraogo E., Mando A., Six J. (2007). Soil fauna and soil function in the fabric of the food web. Pedobiologia, 50(6): 447-462.

6. Brussaard L. (2012). Ecosystem services provided by the soil biota. In: Soil Ecology and Ecosystem Services (eds. D.H. Wall, R.D. Bardgett, V. Behan-Pelletier, J.E. Herrick, T.H. Jones, K. Ritz, J. Six, D.R. Strong\& W.H. van der Putten), pp. 45-58. Oxford University Press, Oxford, UK.

7. Butenschoen O., Marhan S., Langel R., Scheu S. (2009). Carbon and nitrogen mobilisation by earthworms of different functional groups as affected by soils and content. Pedobiologia, 52(4): 263-272.

8. Cesar J., Menaut J.C. (1974). Le peuplement végétal des savanes de Lamto in : Analyse d'un écosystème tropical humide : la savane de Lamto (Côte d'Ivoire). bulletin de liaison des chercheurs Lamto, $\mathrm{N}^{\circ} \mathrm{II}$ : $1-161$.

9. Csuzdi C., Tondoh J.E. (2007). New and little-known earthworm species from the Ivory Coast (Oligochaeta: Acanthodrilidae: Benhamiinae and Eudrilidae). Journal of National History, 41: 25512567.

10. Dewi W.S, Senge M. (2015). Earthworm diversity and ecosystem services underthreat. Rev AgricSci. 3: 25-35.

11. Doan T.T., Jusselme D.M., Lata J.C., Nguyen B.V., Jouquet P. (2013). The earthworm species Metaphireposthuma modulates the effect of organic amendments (compost vs. vermicompost from buffalo manure) on soil microbial properties. A laboratory experiment. European Journal of Soil Biology, 59:15-21.

12. Ehouman N.M, Tiho S., Dagnogo M.(2011). Plant community influences on earthworms in Lamto savannahs (Côte d'Ivoire). Journal of Applied Biosciences, 42: 2841-2853. 
13. Eisenhauer N., Schädler M. (2011). Inconsistent impacts of decomposer diversity on the stability of aboveground and below ground ecosystem functions. Oecologia, 165:403-415.

14. Gobat J.M., Aragno M., Matthey W. (2003). Le sol vivant. Presses Polytechniques et Universitaires Romandes. Lausanne. 568 p.

15. Gobat, J.M., Aragno, M., Matthey, W. (2010). Le sol vivant: bases de pédologie, biologie des sols: PPUR Presses polytechniques.

16. Hasine B.H, Aloui T., Gallali T., Bouzid T., El Amri S, Hassen B.R. (2008). Evaluation quantitative et rôle de la matière organique dans les sols cultivés en zones subhumides et semiarides méditerranéenne de la Tunisie. Agrosolutions, 19(2): 4-17.

17. Iman N. F., Rahmani R. (2005). Relation between number and biomass of earthworms with tree species and soil properties. M.Sc. Thesis, GorganUniversity. $340 \mathrm{p}$.

18. Jégou D., Cluzeau D., Hallaire V., Balesdent J., Trehen P. (2000). Burrowing activity of the earthworms Lumbricus terrestris and Aporrectodea giardi and consequences on $\mathrm{C}$ transfers in soil.European Journal of Soil Biology. 36: 27-34.

19. Laossi K-R., Noguera D.C., Bartolomé-Lasa A., Mathieu J., Blouin M., Barot S. (2009). Effects of endogeic and anecic earthworms on the competition between four annual plants and their relative reproduction potential. Soil Biology and Biochemistry, 41:1668-1673.

20. Lavelle P. (1978). Les vers de terre de la savane de Lamto (Côte d'Ivoire): peuplements, populations et fonctions dans l'écosystème. Doctorat thesis, Université Paris VI, 301 p.

21. Lavelle P. (1983). The soil fauna of tropical savannahs. II. The earthworms. In: Tropical Savannahs. Ed. F. Bourliere. Ecosystems of the World-13. Elsevier. The Netherlands, pp. 485-504.

22. Lavelle P., Spain A.V. (2001). Soil Ecology. Kluwer Scientific Publications, Amsterdam, pp. 201-318.

23. Pelosi C., Bertrand M., Makowski D., Roger-Estrade J. (2008). WORMDYN: A model of Lumbricus terrestris population dynamics in agricultural fields. Ecological Modelling, 218: 219-234.

24. Pérès G., Vandenbulcke F., Guernion M., Hedde M., Beguiristain T., Douay F., Houot S., Piron D., Richard A., Bispo A., Grand C., Galsomies L., Cluzeau D. (2011). Earthworm indicators as tools for soil monitoring, characterization and risk assessment. An example from the national Bioindicator programme (France). Pedobiologia, 54, Supplement: S77-S87.

25. Römbke J., Jansch S., Didden W.(2005). The use of earthworms in ecological soil classification and assessment concepts. Ecotoxicology and Environmental Safety, 62: 249-265. 
26. Satchell J.A. (1980). Worms and K worms. A basis for classifying lumbricidea earthworm strategies. In: Dindal, D.L. (Ed.), SoilBiology as Related to Land Use Practices. Proc. VIIthSoil Zool. Colloq. EPA, Washington, D.C., pp. 848-854.

27. Sautter K.D., Brown C.G., James S.W., Pasini D.H., Nunesand N., Benito P. (2006). Present knowledge on earthworm biodiversity in the state of Parana, Brazil. European Journal of Soil Biology, 42: 296-300. 28. Singh S.P., Singh J., Vig A.P. (2016). Earthworm as ecological engineers to change the physico-chemical properties of soil: Soil vermicast. Ecological engineering, 90:1-5. 